\title{
Synthesis of CdS and CdSe nanoparticles by thermolysis of diethyldithio- or diethyldiseleno-carbamates of cadmium ${ }^{\dagger}$
}

\author{
Tito Trindade and Paul O'Brien* \\ Department of Chemistry, Queen Mary and Westfield College, Mile End Road, London, UK E1 4 NS
}

Cadmium sulfide and cadmium selenide nanoparticles have been synthesised by a novel route involving the thermal decomposition of the bisdiethyldithio- or bisdiethyldiseleno-carbamates of cadmium in refluxing 4-ethylpyridine solutions. The nanodispersed materials were studied by electronic spectroscopy and bandgaps were blue shifted. Transmission electron microscopy of the samples showed material to be in the nanosize range and crystalline.

There has been considerable interest in the synthesis and characterisation of semiconductor nanoparticles. ${ }^{1-5}$ Nanoparticles, also known as nanocrystallites, Q-particles or quantum dots, are particles with a high surface: volume ratio and diameters of up to $10-20 \mathrm{~nm}$, their opto-electronic properties are different from the bulk counterparts, and new technological applications have been proposed for this type of materials..$^{5-7}$ The prospects for devices are now more immediate and a number of recent papers have reported on either the photoluminescent properties of nanodispersed II-VI materials ${ }^{8}$ or photoluminescent devices based on such II-VI materials. ${ }^{9}$ Nanoparticles are also important in fundamental research because they represent a state of matter in which the transition from molecular to the bulk (macrocrystalline) level can be investigated experimentally. ${ }^{1-5}$

The preparations of nanoparticles of many semiconductors have been reported, these include: $\mathrm{PbS},{ }^{10,11} \mathrm{CdS}, 1,11-16$ $\mathrm{CdSe},{ }^{16-19} \mathrm{CdTe},{ }^{16} \mathrm{ZnS},{ }^{20-22} \mathrm{ZnO}^{23} \mathrm{TiO}_{2},{ }^{24} \mathrm{InP},{ }^{25} \mathrm{GaAs},{ }^{26,27}$ $\mathrm{Zn}_{3} \mathrm{P}_{2}{ }^{21}$ and $\mathrm{Cd}_{3} \mathrm{P}_{2} \cdot{ }^{20,21}$ More recently, the synthesis of nanocomposites has been subject of intense research as well. Some examples of nanocomposite materials described in the literature are $\mathrm{ZnS} / \mathrm{CdSe}{ }^{19} \mathrm{CdS} / \mathrm{PbS},{ }^{11,28} \mathrm{SiO}_{2} / \mathrm{CdS}^{29}$ and $\mathrm{CdS} / \mathrm{ZnS}^{30}$ There are other reports of studies concerning the preparation of nanoparticulate systems including elemental $\mathrm{Ag}^{31} \mathrm{Ge},{ }^{32}$ $\mathrm{Pd}^{33}$ and $\mathrm{Pt}^{34}$ or metal halides such as $\mathrm{HgI}_{2}{ }^{35}$ and $\mathrm{PbI}_{2}{ }^{36}$

Theoretical models predicting the optical properties of semiconductor nanoparticles are available $e^{37-41}$ but the properties of nanoparticles obtained by any new synthetic procedure are hard to anticipate. The following characteristics are desirable in the final nanodispersed system: high purity, monodispersity and an ability to control surface derivatization. Nanoparticles with these properties have been prepared by several synthetic methods and/or separation techniques. ${ }^{2,4}$ The chemical methods used for the preparation of semiconductor nanoparticles involve reactions in various media including: aqueous solution, microemulsions, zeolites, gels, polymers and glasses.

Steigerwald et al. ${ }^{18}$ prepared CdSe nanoparticles using the solution-phase thermolysis of $\mathrm{Cd}\left[\operatorname{Se}\left(\mathrm{C}_{6} \mathrm{H}_{5}\right)\right]_{2}$. The method involved ${ }^{18}$ refluxing the precursor in 4-ethylpyridine, a high boiling point solvent, to give optically clear solutions containing the nanoparticles. The high reflux temperature promotes the decomposition of the precursor producing the semiconductor nanoparticulate material. Such single-molecule precursors contain the metal and non-metal (chalcogenide) within the same molecule and are therefore attractive sources for

$\uparrow$ Presented at the Second International Conference on Materials Chemistry, MC $^{2}$, University of Kent at Canterbury, 17-21 July 1995. $\ddagger$ Present address: Department of Chemistry, Imperial College of Science, Technology and Medicine, South Kensington, London, UK SW7 2AZ. the one-step preparations of nanoparticles containing those elements. Such an approach avoids the use of toxic and pyrophoric compounds such as $\mathrm{Cd}\left(\mathrm{CH}_{3}\right)_{2}$, which is commonly used for preparing nanodispersed cadmium chalcogens. ${ }^{16}$

Solid cadmium diethyldithiocarbamate (Cddtc) and cadmium diethyldiselenocarbamate (Cddsc) are dimeric compounds of molecular formula $\left\{\mathrm{Cd}\left[\mathrm{E}_{2} \mathrm{CN}\left(\mathrm{C}_{2} \mathrm{H}_{5}\right)_{2}\right]_{2}\right\}_{2}(\mathrm{E}=\mathrm{S}$, $\mathrm{Se})$. Their crystal structures have been reported ${ }^{42,43}$ and show distorted square-pyramidal coordination at the metal. The bisdiethyldithio- or bisdiethyldiseleno-carbamates of cadmium have been used in chemical vapour deposition experiments to prepare II-VI semiconductor films. ${ }^{42,44}$ In this work, solutions of these compounds in 4-ethylpyridine were used to produce $\mathrm{CdS}$ and CdSe nanoparticles. This solvent has a high boiling point $\left(168^{\circ} \mathrm{C}, 1 \mathrm{~atm}\right)$ and dilute solutions of Cddtc and Cddsc in 4-ethylpyridine remain optically clear for more than $24 \mathrm{~h}$. Nanoparticulate material with a derivatized surface has previously been obtained by using 4-ethylpyridine as the solvent; ${ }^{18}$ however, metal thiocarbamates/selenocarbamates have never been used as precursors for semiconductor nanoparticles.

\section{Experimental}

\section{Chemicals}

$\mathrm{CdCl}_{2}\left(99+\%, \quad\right.$ Aldrich), $\quad \mathrm{NaS}_{2} \mathrm{CN}\left(\mathrm{C}_{2} \mathrm{H}_{5}\right)_{2} \cdot 3 \mathrm{H}_{2} \mathrm{O} \quad(98 \%$, Aldrich), 4-ethylpyridine (98\%, Aldrich), pyridine $(99+\%$, Aldrich, $\mathrm{CH}_{2} \mathrm{Cl}_{2}(99 \%, \mathrm{BDH})$ and light petroleum (bp $60-80^{\circ} \mathrm{C}, \mathrm{BDH}$ ) were all used as received except 4-ethylpyridine which was dried with molecular sieves (type $3 \AA$, BDH) and deoxygenated under a nitrogen flow.

\section{Synthesis of molecular precursors}

Cddtc was synthesized by adding stoichiometric quantities of aqueous equimolar $\left(0.1 \mathrm{~mol} \mathrm{dm}{ }^{-3}\right)$ solutions of $\mathrm{CdCl}_{2}$ and $\mathrm{NaS}_{2} \mathrm{CN}\left(\mathrm{C}_{2} \mathrm{H}_{5}\right)_{2} \cdot 3 \mathrm{H}_{2} \mathrm{O}$. The white solid obtained was filtered off and washed thoroughly with deionised water. This solid was purified by recrystallization from hot $\mathrm{CH}_{2} \mathrm{Cl}_{2}$. Cddsc was synthesized by the method described in the literature ${ }^{42}$ by treating $N, N$-diethyldiselenocarbamate, as the diethylammonium salt, with an aqueous solution containing a stoichiometric amount of $\mathrm{CdCl}_{2}$. The compounds were identified by ${ }^{1} \mathrm{H}$ NMR $\left(\mathrm{CDCl}_{3}\right)$ and IR spectroscopy.

\section{Synthesis of the CdS and CdSe nanoparticles}

Solutions (5-50 mmol dm $\mathrm{dm}^{-3}$ in the precursor) were prepared by dissolving the required amount of the compound in 4ethylpyridine at room temperature. The solutions were filtered and then heated at the reflux temperature of 4-ethylpyridine 
$\left(168^{\circ} \mathrm{C}\right)$. The reflux was performed both under the ambient atmosphere and an $\mathrm{N}_{2}$ atmosphere. The formation of the nanoparticles as a function of the time of heating was monitored by extracting an aliquot of the refluxing solutions and transferring it to a vial immersed in an ice-bath and recording the optical absorption spectrum. The addition of light petroleum to the final cooled and optically clear solutions resulted in the flocculation of a precipitate which was collected by centrifugation. These solids were washed with dichloromethane-light petroleum and then dried under vacuum to give powders which were stored under $\mathrm{N}_{2}$. The material gave optically clear solutions when redissolved in either pyridine or 4-ethylpyridine; any insoluble material in these redispersions was isolated by centrifugation and discarded.

\section{Material characterisation and instrumentation}

IR spectroscopy of the powders was performed using CsI (99.9\%, Aldrich) pellets and a Perkin-Elmer 1720X FTIR spectrometer. Optical absorption spectra were recorded at room temperature with a Philips PU 8710 spectrophotometer. Silica cells $(1 \mathrm{~cm})$ were used and the starting solution for each precursor was used as reference. The pyridine solutions were analysed using pure pyridine as reference. The ${ }^{1} \mathrm{H}$ NMR spectra were recorded in a Bruker $250 \mathrm{AM}$ pulsed Fourier transform instrument.

Scanning electron microscopy (SEM) and energy dispersive X-ray (EDAX) spectroscopy were performed with a JEOL JSM35CF microscope operating at $25 \mathrm{kV}$. The samples for analysis were prepared by putting one drop of the sample solution onto pure aluminium plates and letting the solvent evaporate at room temperature.

X-Ray powder diffraction (XRPD) patterns were measured using a Guinier camera and a Philips $1130 \mathrm{X}$-ray generator ( $\mathrm{Cu}-\mathrm{K} \alpha$ radiation). Samples for $\mathrm{X}$-ray diffraction were prepared by placing the powder onto adhesive tape.

Conventional transmission electron microscopy (TEM) results were obtained using a JEOL-JEM 1200 EX II scanning and transmission electron microscope operating at $100 \mathrm{kV}$; high resolution transmission electron microscopy (HRTEM) was performed with a JEOL $2000 \mathrm{FX}$ electron microscope operating at $200 \mathrm{kV}$. A sample for TEM was prepared by placing an aliquot of pyridine solution containing the nanoparticles onto an amorphous carbon surface on a copper grid and wicking away the solvent with a paper tip. Particle sizes were determined by measuring the diameter of around one thousand particles on the TEM images.

\section{Results and Discussion}

\section{Optical properties of nanodispersed CdS and CdSe}

The optical properties of solutions of Cddtc and Cddsc in 4ethylpyridine change with heating. Typical changes, as a function of time of heating, using the starting solution as reference, are reported in Fig. 1 and 2 for the Cddtc and Cddsc precursors, respectively. With longer heating times the optical homogeneity of the solutions is not maintained and scattering perturbs the absorption spectra. In both cases the absorption edge is blueshifted in relation to the bulk bandgap value, (the absorption edge is taken as the intersection of the base line with the tangent drawn to the band shoulder). Such shifts in the absorption edge of semiconducting materials have been associated ${ }^{1-5}$ with particles having sizes comparable to the de Broglie wavelength of the electron and hole.

The alterations observed in the band profiles (Fig. 1 and 2) are associated with a chemical transformation of the molecular precursors, since for both cases the starting solution was used as reference. A blank consisting of pure 4-ethylpyridine, refluxed over $6 \mathrm{~h}$ under ambient atmosphere, showed an increase in the intensity of the absorption band at $325 \mathrm{~nm}$. If

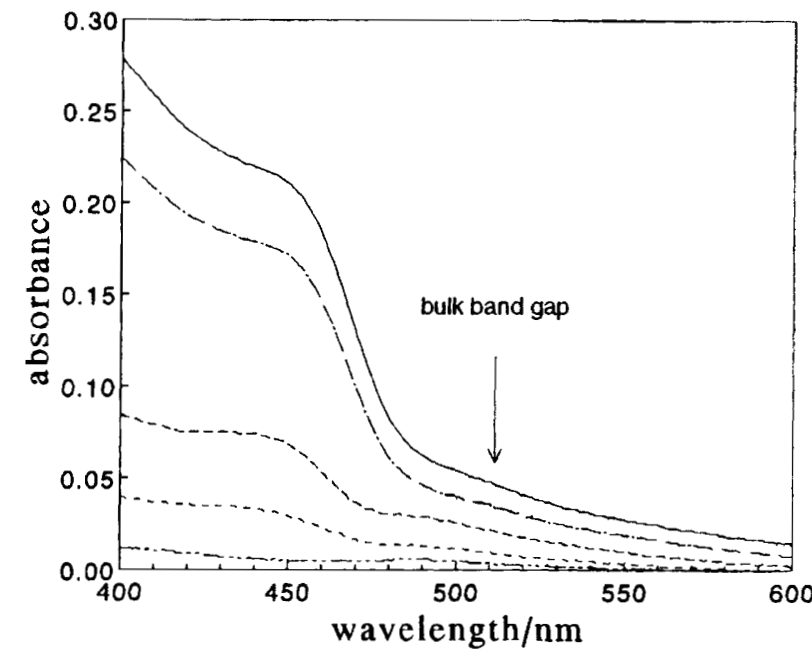

Fig. 1 Optical absorption spectra of Cddtc in 4-ethylpyridine solutions at different reflux times: $-\cdots-, 0.25 ;---, 1 ;----, 2 ; \cdots \cdots, 4$; $-\cdot-\cdot-, 5 ;-, 6 \mathrm{~h}$

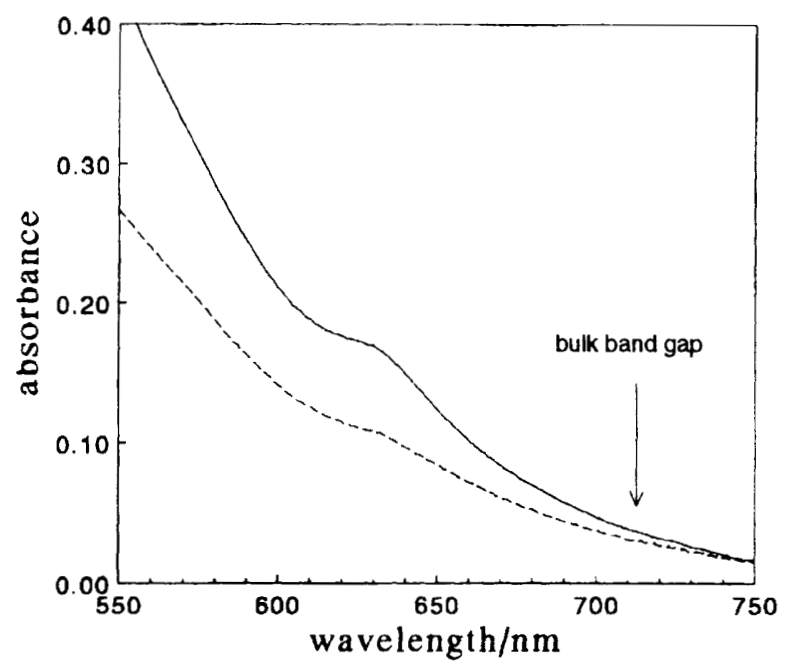

Fig. 2 Optical absorption spectra of Cddsc in 4-ethylpyridine solutions at different reflux times:,$--- 2 ; \cdots \cdots, 4 ;-, 6 \mathrm{~h}$

the reflux is carried out under $\mathrm{N}_{2}$ the 4-ethylpyridine shows no such changes in its optical characteristics. However, the changes associated with the formation of nanodispersed material are similar if heating is performed in the presence or absence of $\mathrm{N}_{2}$. The absorption characteristics for CdS (Fig. 1) and CdSe (Fig. 2) are in agreement with the initial formation of CdS or CdSe nanoparticles which agglomerate to form particles of larger dimensions. Other authors ${ }^{18}$ have reported similar optical spectra for CdSe particles grown from $\mathrm{Cd}\left[\mathrm{Se}\left(\mathrm{C}_{6} \mathrm{H}_{5}\right)\right]_{2}$ in 4-ethylpyridine solutions. The band at $420 \mathrm{~nm}$ was assigned ${ }^{18}$ to electronic transitions occurring in small CdSe clusters dispersed in 4-ethylpyridine. In this work an absorption band at around $413 \mathrm{~nm}$ was also observed in both 4-ethylpyridine and pyridine solutions containing the CdSe species (Fig. 3). The optical absorption spectrum of CdS nanoparticles have been published ${ }^{14,15}$ and are similar to those shown in Fig. 1, even though media as diverse as zeolites ${ }^{14}$ and water ${ }^{15}$ have been used in the preparation.

The species responsible for the absorption were isolated as powders by addition of light petroleum to the cooled solutions followed by centrifugation. The solids obtained are readily dispersed in pure 4-ethylpyridine or pyridine. The optical absorption spectra for the pyridine solutions were recorded, and showed that the shifts in the edges to higher energies compared to the bulk values are still observed (Fig. 3). The 


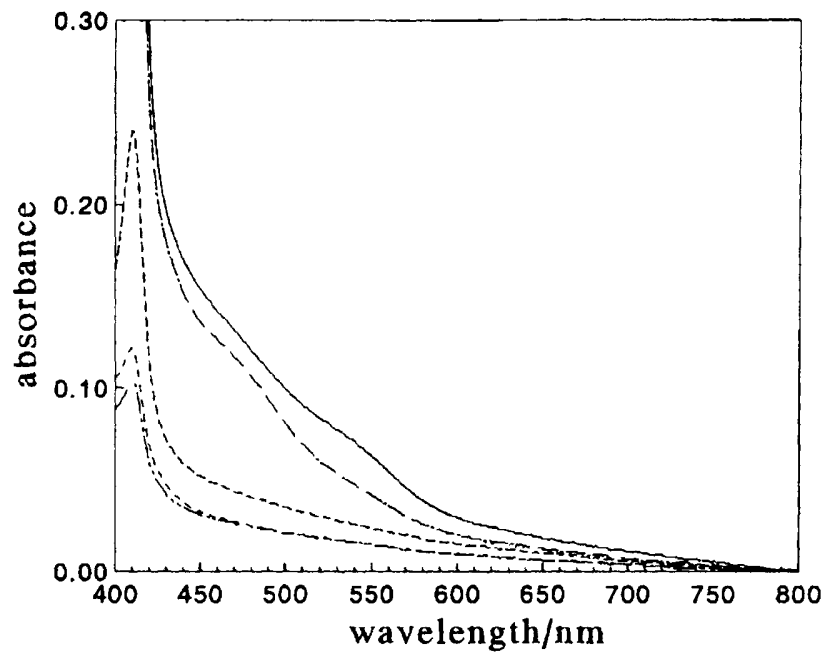

Fig. 3 Optical absorption spectra of the pyridine solutions containing the soluble powders obtained from Cddsc in 4-ethylpyridine at different reflux times: $-\cdots, 0.25 ;-\cdots, 0.5 ;---, 2 ; \cdots \cdots, 4 ;-\cdots \cdot, 5 ;-, 6 \mathrm{~h}$

observed band shifts, on reffuxing, suggest an increase in mean particle diameters for the CdSe clusters with time. ${ }^{16}$

It was found that the pyridine solutions containing the nanoparticles were unstable and the optical properties changed with time. A broadening of the sharp band at $413 \mathrm{~nm}$, after $48 \mathrm{~h}$, can be seen clearly (Fig. 4) for the sample obtained from Cddsc after 15 min reflux. The 4-ethylpyridine growth solutions also shows broadening of the absorption bands when kept standing. Such broadening is to be expected if an agglomeration process occurs because the polydispersity of the particulate system will be increased.

The spectrum of a pyridine solution containing the powder obtained from the addition of light petroleum to the 4ethylpyridine solution of Cddtc after $6 \mathrm{~h}$ reflux is shown in Fig. 5. The 'bandgap' of the $\mathrm{CdS}$ nanoparticles was determined using the direct transition method ${ }^{45}$ by fitting the absorption data to eqn. (1) (Fig. 5, inset):

$$
\alpha(h v) \propto\left(h v-E_{\mathrm{g}}\right)^{1 / 2}
$$

where $\alpha$ is the absorption coefficient of the semiconductor material, $h v$ is the photon energy and $E_{\mathrm{g}}$ is the optical bandgap. The optical bandgap obtained by using this method is $2.63 \mathrm{eV}$, which is slightly blue-shifted from that of bulk CdS $(2.53 \mathrm{eV})$. CdS particles begin to present ${ }^{4}$ the characteristic bandgap of bulk material at a diameter of around $80 \AA$, i.e. for particles within the nanosize range.

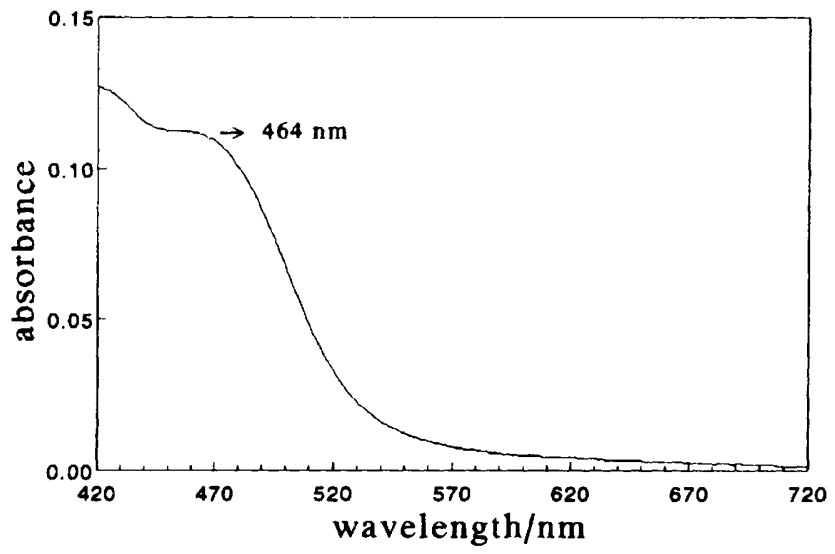

Fig. 4 Optical absorption spectrum of the pyridine solution after $48 \mathrm{~h}$ $(t=0.25$ h in Fig. 3$)$

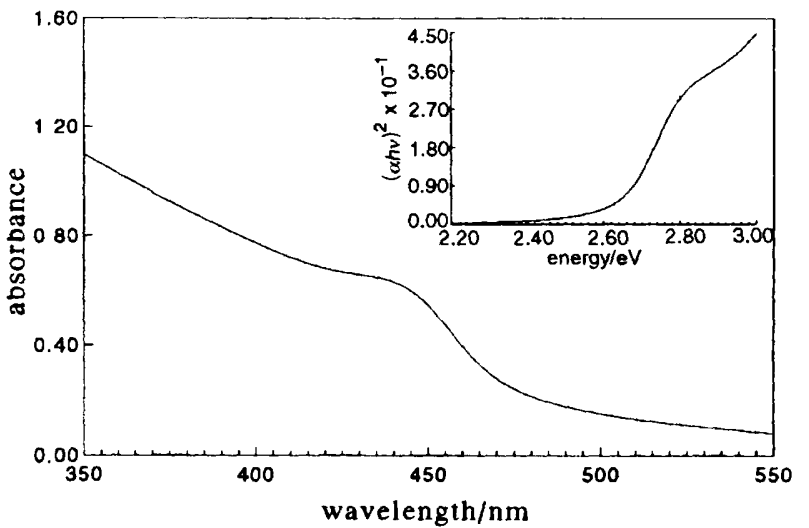

Fig. 5 Optical absorption spectrum of the pyridine solution containing the soluble powder obtained from Cddtc in 4-ethylpyridine ( $6 \mathrm{~h}$ reflux). Inset shows the fit of the absorption edge by the direct transition method.

\section{Characterization of solid phases}

Prolonged times of heating led to 4-ethylpyridine solutions containing solid material in suspension and/or fixed to the walls of the flask. With the dithiocarbamate the suspended material is dark yellow and the XRPD pattern consists of broad lines typical of hexagonal CdS. The bulk material obtained from the selenocarbamate precursor is brown-grey and adhered firmly to the walls of the flask forming a specular film. The XRPD pattern showed evidence for elemental hexagonal Se and hexagonal CdSe; for the latter case the SEM showed well defined spherical particles within the submicrometric range (Fig. 6). EDAX on a single particle showed the presence of both Se and $\mathrm{Cd}$. These results suggest that Cddtc and Cddsc are thermally decomposed in 4-ethylpyridine solutions. Prolonged heating times lead to bulk material but the solutions still contain nanosized particles of $\mathrm{CdS}$ and $\mathrm{CdSe}$, as indicated by their optical absorption spectra. This hypothesis is also supported by the results obtained from the characterisation of the solid phases obtained from the syntheses.

The powders isolated from the solutions during the growth of nanodispersed material were characterised by IR, EDAX and XRPD. The IR spectra of the powders do not show the characteristic bands of the molecular precursors. The ease of dissolution of these powders in pyridine and 4-ethylpyridine suggests the binding of solvent molecules to the nanoparticles surface. However, the characteristic bands of the 4-ethylpyridine were not found in the IR spectra (e.g. the sharp and strong bands due to the ring stretching of 4-ethylpyridine around 1602 and $1560 \mathrm{~cm}^{-1}$ ). It is probable that surface coverage has occurred to an extent below the detection limits of the IR experiment. The low surface coverage of the nanopart-

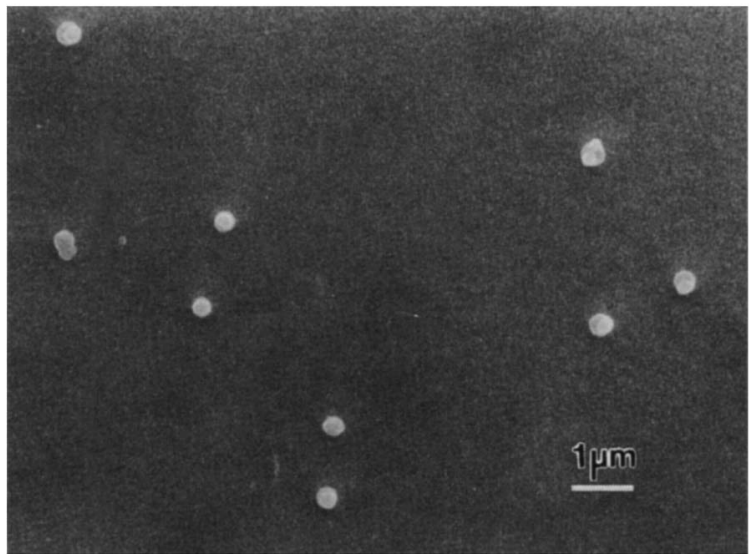

Fig. 6 SEM image of CdSe particles

J. Mater. Chem., 1996, 6(3), 343-347 
(a)

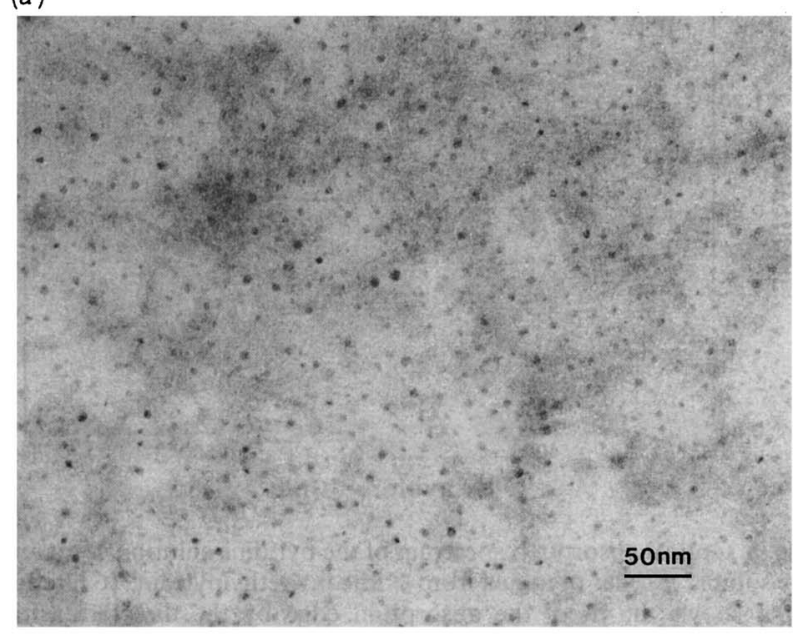

(b)

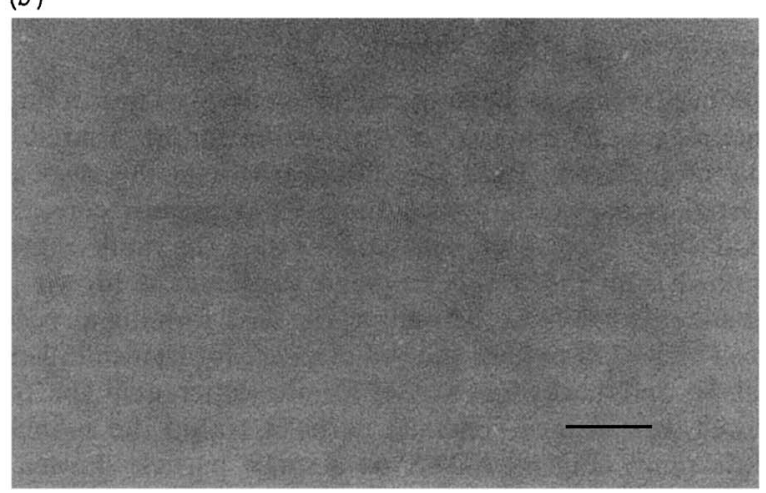

Fig. $7 \mathrm{CdSe}$ nanoparticles obtained from Cddsc in 4-ethylpyndine after refluxing for 5 min (a) conventional TEM image, (b) HRTEM 1mage $($ bar $=10 \mathrm{~nm})$ icles by the solvent molecules could also explain their relatively facile agglomeration

The elements detected by EDAX were $\mathrm{Cd}$ and the chalcogenide element ( $\mathrm{S}$ or $\mathrm{Se}$ ) $\mathrm{S} 1$ and $\mathrm{Cl}$ were also detected as contaminants, probably from vacuum grease and/or precursor Unlıke the bulk solids obtained in both syntheses these powders did not show XRPD patterns in our equipment, a result that does not preclude some crystallinity in the samples

\section{Particle size assessment}

The material contained in the optically clear pyridine solutions containing CdSe was subjected to further analysis by TEM The TEM analysis was performed for samples obtained after 15 min [Fig 7(a), Fig 7(b) shows the HRTEM image] and 360 min reflux The particle size distribution for the shorter time is shown in Fig 8, in which the mean diameter is $48 \AA$ The TEM of a sample refluxed for longer tıme shows larger particles with some agglomeration having occurred

The results obtained by TEM confirm that the agglomeration is a favourable process in the pyridine solutions containing the CdSe nanoparticles The agglomeration leads to some spread on the particle size distribution (Fig 8) In Fig $7(b)$ the lattıce fringes of particles with diameters up to $50 \AA$ are clearly observed, confirming the presence of dispersed nanocrystallites in the pyridine solution Analysis of the patterns for several different particles was most consistent with a predominance of the hexagonal phase This result shows that the CdSe nanopart1cles have the bulk unit-cell structure, despite their markedly different optical properties, in agreement with reports made by other authors 1618

This work showed that single-molecule precursors such as Cddtc and Cddsc can be used for the preparation of soluble nanosized CdS and CdSe particles, respectively Work is in progress in our laboratories on the use of alkyl derivatives ${ }^{46}$ of the precursors used in the work described here Our main concern is to overcome some of the limitations found in this work, such as the practical manipulation of significant quantities of semiconductor nanoparticles and the inherent

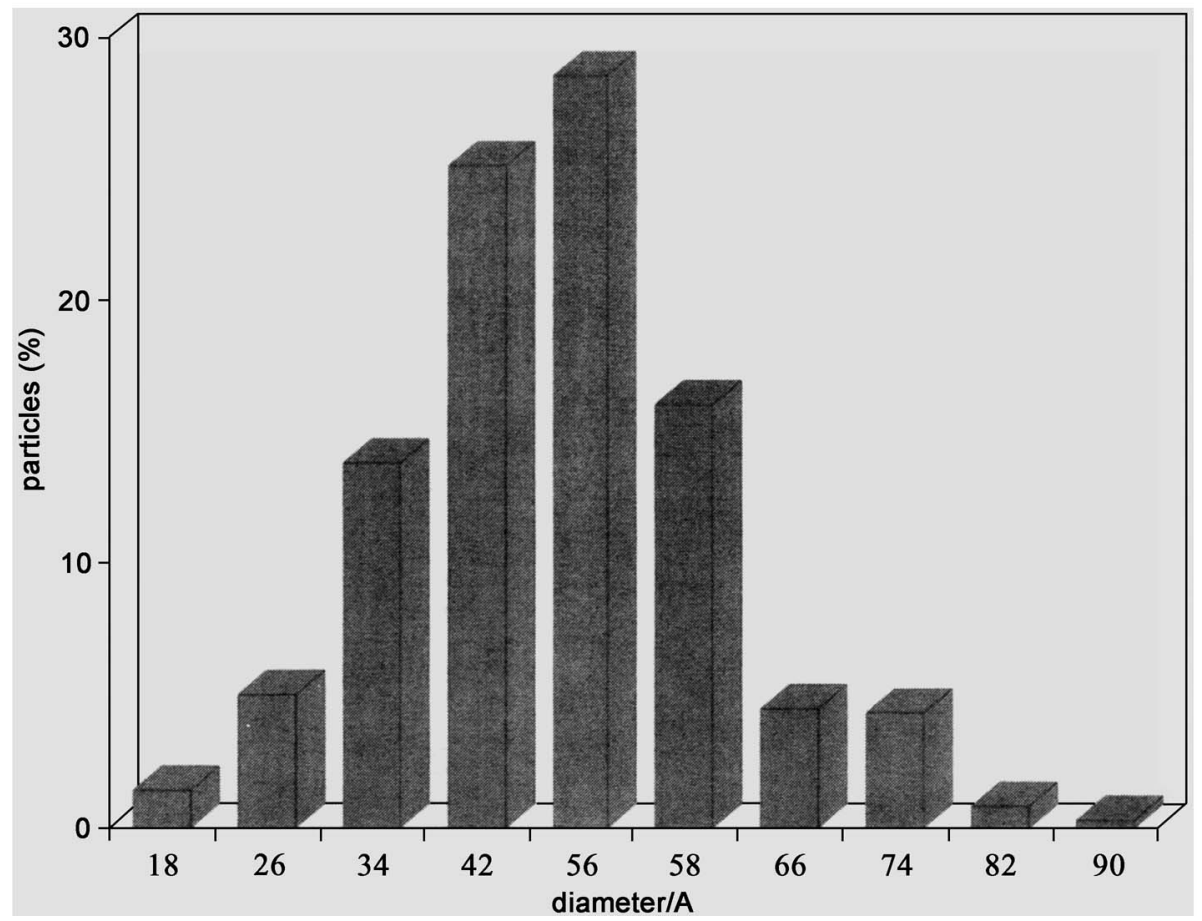

Fig. 8 Partıcle size distribution of the CdSe nanopartıcles obtained from Cddsc in 4-ethylpyridine after refluxing for 15 min and redispersıon in pyridine 
instability of the solutions containing the nanoparticulate materials.

Tito Trindade thanks JNICT (Portugal) for a grant. We thank Mr. K. Pell (QMW College) and Dr. X. Zhang (IRC for Semiconductors, Imperial College) for technical assistance with the microscopy work.

\section{References}

1 R. Rosseti, J. L. Ellison, J. M. Gibson and L. E. Brus, J. Chem. Phys., 1984, 80, 4464.

2 A. Henglein, Chem. Rev, 1989, 89, 1861.

3 M. L. Steigerwald and L. Brus, Acc. Chem. Res., 1990, 23, 183.

4 H. Weller, Angew. Chem., Int. Ed. Engl., 1993, 32, 41.

5 H. Weller, Adv. Mater., 1993, 5, 88

6 L. Brus, J. Phys. Chem., 1994, 98, 3575.

7 A. Fojtik and A. Henglein, Chem. Phys. Lett., 1994, 221, 363.

8 B. O. Dabbousi, C. B. Murray, M. F. Rubner and M. G. Bawendi, Chem. Mater., 1994, 6, 216.

9 V. L. Colvin, M. C. Schlamp and A. P. Alivisatos, Nature, 1994, 370, 354.

10 A. J. Nozik, F. Williams, M. Nenadovic, T. Rajh and O. I. Micic, J. Phys. Chem., 1985, 89, 397.

11 H. S. Zhou, I. Honma, H. Kumiyama and J. W. Haus, J. Phys. Chem., 1993, $97,895$.

12 A. Fojtik, H. Weller, U. Koch and A. Henglein, Ber. Bunsenges. Phys. Chem., 1984, 88, 969.

13 L. Spanhel, M. Haase, H. Weller and A. Henglein, J. Am. Chem. Soc., 1987, 109, 5649 .

14 Y. Wang and N. Herron, J. Phys. Chem., 1987, 91, 257.

15 H. J. Watzke and J. H. Fendler, J. Phys. Chem., 1987, 91, 857

16 C. B. Murray, D. J. Norris and M. G. Bawendi, J. Am. Chem. Soc, $1993,115,8706$.

17 M. L. Steigerwald, A. P. Alivisatos, J. M. Gibson, T. D. Harris, R. Kortan, A. J. Muller, A. M. Thayer, T. M. Duncan, D. C. Douglas and L. E. Brus, J. Am. Chem. Soc., 1988, 110, 3046.

18 J. G. Brenman, T. Siegrist, P. J. Carroll, M. Stuczynski, L. E. Brus and M. L. Steigerwald, J. Am. Chem. Soc., 1989, 111, 4141.

19 A. R. Kortan, R. Hull, R. L. Opila, M. G. Bawendi, M. L Steigerwald, P. J. Carrol and L. E. Brus, J. Am. Chem. Soc., 1990, $112,1327$.

20 S. Baral, A. Fojtik, H. Weller and A. Henglein, J. Am. Chem. Soc., 1986, 108, 375 .
21 H. Weller, A. Fojtik and A. Henglein, Chem. Phys. Lett., 1985, $117,485$.

22 V. Sankaran, J. Yue, R. E. Cohen, R. R. Schrock and R. J. Silbey, Chem. Mater., 1993, 5, 1133.

23 A. J. Hoffman, H. Yee, G. Mills and M. R. Hoffmann, J. Phys. Chem., 1992, 96, 5540.

24 D. Duoghong, J. Ramsden and M. Grätzel, J. Am. Chem. Soc., 1982, 104, 2977.

25 T. Douglas and K. H. Theopold, Inorg. Chem., 1991, 30, 594.

26 M. A. Olshavsky, A. N. Goldstein and A. P. Alivisatos, J. Am. Chem. Soc., 1990, 112, 9438.

27 P. C. Sercel, W. A. Saunders, H. A. Atwater, K. J. Vahala and R. C. Flagan, Appl. Phys. Lett., 1992, 61, 696.

28 H. S. Zhou, H. Sasahara, I. Honna, H. Komiyama and J. W. Haus, Chem. Mater., 1994, 6, 1534.

29 S. Chang, L. Liu and S. A. Asher, J. Am. Chem. Soc., 1994, 116, 6739.

30 T. Cassagneau, G. B. Hix, D. J. Jones, P. Meireles-Torres, M. Rhomi and J. Roziere, J. Mater. Chem., 1994, 4, 189.

31 A. Henglein, P. Mulvaney and T. Linnert, Faraday Discuss., 1991, 92, 31 .

32 A. Kornowski, M. Giersig, R. Vogel, A. Chemseddine and H. Weller, Adv. Mater., 1993, 5, 634.

33 C. Amiens, D. Caro, B. Chautret and J. S. Bradley, J. Am. Chem. Soc., 1993, 115, 11638 .

34 M. T. Reetz and W. Helbig, J. Am. Chem. Soc., 1994, 116, 7401

35 M. W. Peterson, O. I. Micic and A. J. Nozik, J. Phys. Chem., 1988, 92, 4160.

36 O. I. Micic, L. Zonggnem, G. Mills, J. C. Sullivan and D. Meisel, J. Phys. Chem., 1987,91, 6221.

37 L. E. Brus, J. Chem. Phys., 1983, 79, 5560

38 L. E. Brus, J. Chem. Phys., 1984, 80, 4403.

39 P. E. Lippens and M. Lannoo, Phys. Rev. B, 1989, 39, 10395.

40 Y. Wang and N. Herron, J. Phys. Chem., 1991, 95, 525.

41 Y. Nosaka, J. Phys. Chem., 1991, 95, 5054

42 M. B. Hursthouse, M. Azad Malik, M. Motevalli and P. O'Brien, Polyhedron, 1992, 11, 45.

43 M. Bonamico, G. Mazzone, A. Vaciago and L. Zambonelli, Acto Crystallogr., 1965, 19, 898

44 D. M. Frigo, O. F. Z. Khan and P. O'Brien, J. Crystal Growth, $1989,96,989$

45 I. Pankove, Optical Processes in Semiconductors, Dover Publications, New York, 1970, p. 36.

46 M. Azad Malik and P. O'Brien, Adv. Mater. Opt. Electron., 1994, 3,171 .

Paper 5/05017K; Received 28th July, 1995 